\title{
Development of a Numerical Model Designed to Calculate the Temperature Field and Thermal Stresses in Structural Elements of Aircrafts
}

\author{
V.V. Kuzenov ${ }^{1,2}$, V.V. Shumaev², A.O. Dobrynina² \\ ${ }^{1}$ Dukhov Research Institute of Automatics (VNIIA), \\ Moscow, 127055, Russia \\ ${ }^{2}$ Bauman Moscow State Technical University, \\ Moscow, 105005, Russia \\ vik.kuzenov@gmail.com; chubchic@gmail.com
}

\begin{abstract}
The algorithm for the numerical solution of the quasistatic thermoelasticity problem in the domains of simple spatial forms is briefly described. The initial validation and verification of the developed numerical technique was performed. Some results of the solution of the quasistatic thermoelasticity problem in the simplest structural elements of aircraft.
\end{abstract}

Keywords: mathematical model, gas dynamics, boundary layer, effective length

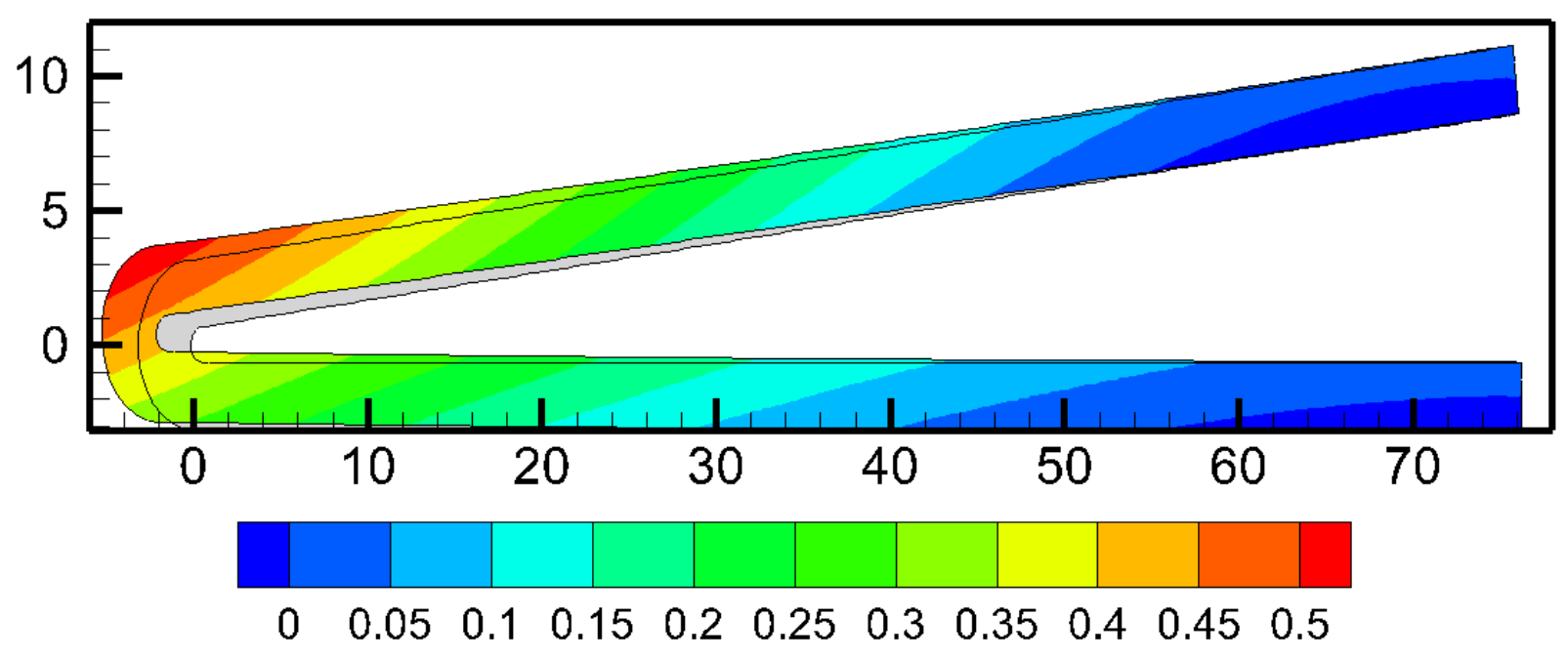

The distribution of displacements $U_{y}[\mathrm{~mm}]$ in the $Y$-axis direction in a wedge-shaped shell (Inconel 617 alloy) blunt in the cylinder. The radius of blunting of the cylinder is $R=3.18 \mathrm{~mm}$, the Mach number in the oncoming stream is $M=5.25$. Solid lines indicate the outlines of the original undeformed body 


\title{
Численная методика расчета температурного поля и термонапряжений в элементах конструкций летательных аппаратов
}

\author{
В.В. Кузенов ${ }^{1,2}$, В.В. Шумаев ${ }^{2}$, А.О. Добрынина ${ }^{2}$ \\ ${ }^{1}$ Федеральное государственное унитарное предприятие «Всероссийский научно- \\ исследовательский институт автоматики им. Н.Л. Духова», \\ Россия, Москва, 127055, ул. Сушеевсккая, д. 22 \\ ${ }^{2}$ МГТУ им. Н.Э. Баумана, Россия, Москва, 105005, 2-я Бауманская ул., д. 5, стр. 1 \\ vik.kuzenov@gmail.com; chubchic@gmail.com
}

\begin{abstract}
Аннотация
Кратко описан алгоритм численного решения квазистатической задачи термоупругости в областях простых пространственных форм. Выполнена первоначальная валидация и верификация разработанной численной методики. Приведены отдельные результаты решения квазистатической задачи термоупругости в простейших элементах конструкций летательных аппаратов.
\end{abstract}

Ключевые слова: математическая модель, газодинамика, пограничный слой, квазистатическая задача термоупругости, эффективная длина

\section{1. Введение}

Одной из наиболее сложных задач аэротермодинамики является задача моделирования сопряженного теплообмена на поверхности высокоскоростного летательного аппарата (ЛА), а также расчет, возникающих вследствие этого термонапряжений в его конструкции. В этом случае движения ЛА пограничный слой можно рассматривать, как узкую пространственную область, примыкающую к поверхности обтекаемого тела в которой происходит интенсивное выделение теплоты за счет процессов диссипации энергии. Процессы диссипации сопровождаются сильным изменением теплофизических (плотность, давление, температура, вязкость, теплопроводность и т.д.), динамических свойств газа, тепловых потоков, направленных к поверхности летательного аппарата, материала конструкции и рабочих характеристик. Однако, проведение реальных физических экспериментов в рассматриваемой области движения ЛА отличается высокой стоимостью, связано с множеством технологических и технических трудностей. Поэтому большое значение для оптимизации рабочих характеристик и конструкции летательного аппарата имеет математическое моделирование термонапряжений и теплофизических процессов в ЛА, а также вблизи его поверхности.

Целью данной работы является разработка приближенного метода оценки термонапряжений в ЛА (или в их ключевых элементах: кромках корпуса и крыльев, носовом обтекателе) простой пространственной формы, например, в форме сферы, сопряженной с цилиндром. Математическая модель оценок, предназначенная для расчета термонапряжений в отдельных элементах конструкции летательных аппаратов, опирается на решение квазистатической задачи термоупругости [1-5]. Она включает в себя уравнения механического равновесия линейно-упругой среды с учетом температурных напряжений (однако эта математическая модель не позволяет проводить полное описание термомеханических процессов, так как в ней отсутствует основные физические механизмы, учитывающие пластическое деформирование конструкционных материалов) и уравнение теплопроводности специаль- 
ного вида. Как правило, физическая область (в которой ищется решение) имеет криволинейные границы, а их форма в процессе моделирования изменяются под действием тепловых и механических нагрузок. Для решения задачи с такими пространственными границами расчетная область преобразуется к области, в которой возможно введение регулярной декартовой (структурированной) сетки.

При оценке термонапряжений в ЛА вводятся следующие, упрощающие предположения:

- $\quad$ принимается, что конструкционный материал является изотропной сплошной средой;

- $\quad$ геометрия и теплонапряженное состояние конструкционного элемента ЛА описывается в двумерной $(2 D)$ постановке;

- $\quad$ изначально задаются внешние механические (силы, поля давлений) и тепловые воздействия (конвективный и радиационный тепловые потоки);

- $\quad$ считается, что скорость акустических волн в конструкционных элементах летательных аппаратов существенно больше скорости распространения в них тепловых волн.

\section{2. Математическая постановка задачи оценки термонапряжений в элементах конструкции летательных аппаратов}

Математическая постановка рассматриваемой задачи сводится к численному решению системы двумерных уравнений квазистатической термоупругости, которые позволяют определить поля температур $T$ и перемещений $\vec{U}$, компоненты тензоров напряжений $\varepsilon_{i j}$ и деформаций $\sigma_{i j}$ в конструкционных элементах ЛА [2-8]

$$
\begin{gathered}
\mu \operatorname{div}(\operatorname{grad}(\vec{U}))+(\lambda+\mu) \operatorname{grad}(\operatorname{div} \vec{U})-(3 \lambda+2 \mu) \alpha_{T} \operatorname{grad}(\theta)=0, \\
\rho c_{\varepsilon=0} \frac{\partial \theta}{\partial t}+\alpha_{T}(3 \lambda+2 \mu) T_{0} \operatorname{div}(\partial \vec{U} / \partial t)=\operatorname{div}\left(\lambda_{q} \operatorname{grad} \theta\right),
\end{gathered}
$$

где $t, x_{j}$ - время и декартовы координаты; $\vec{U}$ - вектор, описывающий перемещение точки тела; $T$ - температура тела; $T_{0}$ - исходная (начальная) температура тела; $\theta=T-T_{0}-$ избыточная температура; $\rho$ - плотность в теле; $c_{\varepsilon=0}$ - теплоемкость при нулевой деформации; $\mu, \lambda-$ коэффициенты Ляме; $\alpha_{T}-$ коэффициент термического расширения.

Граничные условия для температуры формулируются путем задания теплового потока на поверхности элемента конструкции ЛА

$$
-\left.\lambda_{q} \frac{\partial T}{\partial x_{i}}\right|_{\Gamma}=q_{i},
$$

где $T_{0 i}, q_{i}$ - заданные константы; символом Г обозначается поверхность ЛА. Механические граничные условия в виде напряжений задаются аналогично работе [3]. Начальные условия (для момента времени $t=0$ ) определяются в виде изначально заданного пространственного распределения температуры $T$ и перемещений $\vec{U}$.

Граничные условия, необходимые для решения квазистатической задачи термоупругости конечно-разностным методом, удобно реализуются, когда границы расчетной области $\Omega$ совпадают с координатными линиями в некой обобщенной системе координат $\xi, \eta$. При этом расчетная область $\Omega$ переходит в параметрическую область $\Omega_{p}$ (например: в прямоугольник).

Введем преобразование координат вида

$$
r=r(\xi, \eta), z=z(\xi, \eta)
$$


При известных в физическом пространстве координатах узлов сетки в расчетной области $\xi, \eta$ метрические коэффициенты в общем случае могут быть найдены путем численного дифференцирования по формулам

$$
\begin{gathered}
J=\frac{\partial(r, z)}{\partial(\xi, \eta)}=\frac{\partial r}{\partial \xi} \frac{\partial z}{\partial \eta}-\frac{\partial r}{\partial \eta} \frac{\partial z}{\partial \xi} \\
\xi_{r}=J^{-1} \frac{\partial z}{\partial \eta}, \quad \eta_{r}=-J^{-1} \frac{\partial z}{\partial \xi}, \quad \xi_{z}=-J^{-1} \frac{\partial r}{\partial \eta}, \quad \eta_{z}=J^{-1} \frac{\partial r}{\partial \xi},
\end{gathered}
$$

где $J=\partial(r, z) / \partial(\xi, \eta)$ - якобиан перехода от цилиндрической системы координат $r, z$ к криволинейной системе координат $\xi, \eta$.

Использование в уравнениях линейной теории упругости и уравнение теплопроводности эйлеровых координат приводит к возможности построения экономичных, алгоритмически простых разностных схем (за счет упрощения по сравнению лагранжевым подходом численной аппроксимации пространственных производных), дающих возможность сквозного расчета течений с сильными пространственными деформациями.

Для поиска функций $r(\xi, \zeta), z(\xi, \zeta)$ может быть использована система уравнений, полученная в работе [9]. Данные уравнения гарантируют, что найденные функции $r=r(\xi, \eta), z=z(\xi, \eta)$ являются гладкими функциями координат $\xi, \eta$. Однако в некоторых случаях созданная таким образом расчетная сетка по тем или иным причинам может быть признана неудачной. В этой ситуации (вместе с дифференциальными способами) целесообразно использовать аналитические алгебраические преобразования [10].

Система уравнений термоупругости (1) в векторной полудивергентной форме в произвольной криволинейной системе координат $\xi, \eta$ имеет следующий вид:

$$
A \vec{U}=B \frac{\partial \vec{F}}{\partial \xi}+B \frac{\partial \vec{G}}{\partial \eta}+C_{\xi} \frac{\partial \vec{I}}{\partial \xi}+C_{\eta} \frac{\partial \vec{I}}{\partial \eta}+D_{\xi} \frac{\partial \vec{W}}{\partial \xi}+D_{\eta} \frac{\partial \vec{W}}{\partial \eta}+\vec{S}=0
$$

Векторы, входящие в данную систему уравнений, записываются в следующем виде:

$$
\begin{aligned}
& \vec{F}=\left\|\begin{array}{l}
F_{1} \\
F_{2}
\end{array}\right\|, \vec{G}=\left\|\begin{array}{l}
G_{1} \\
G_{2}
\end{array}\right\|, \vec{I}=\|I\|, \vec{W}=\left\|\begin{array}{l}
\theta \| \\
\theta
\end{array}\right\|, \\
& B=\left[\begin{array}{cc}
\frac{\mu}{J} & 0 \\
0 & \frac{\mu}{J}
\end{array}\right], C_{\xi}=\left[\begin{array}{cc}
(\lambda+\mu) \xi_{r} & 0 \\
0 & (\lambda+\mu) \xi_{z}
\end{array}\right], C_{\eta}=\left[\begin{array}{cc}
(\lambda+\mu) \eta_{r} & 0 \\
0 & (\lambda+\mu) \eta_{z}
\end{array}\right], \\
& D_{\xi}=\left[\begin{array}{cc}
\alpha_{T}(3 \lambda+2 \mu) \xi_{r} & 0 \\
0 & \alpha_{T}(3 \lambda+2 \mu) \xi_{z}
\end{array}\right], D_{\eta}=\left[\begin{array}{cc}
\alpha_{T}(3 \lambda+2 \mu) \eta_{r} & 0 \\
0 & \alpha_{T}(3 \lambda+2 \mu) \eta_{z}
\end{array}\right] \text {, } \\
& I=\operatorname{div} \vec{U}=\left[\frac{1}{J} \frac{\partial J\left(U_{\xi}\right)}{\partial \xi}+\frac{1}{J} \frac{\partial J\left(U_{\eta}\right)}{\partial \eta}\right] \text {, } \\
& F_{1}=J\left(\xi_{r}^{2}+\xi_{z}^{2}\right) u_{\xi}, F_{2}=J\left(\xi_{r}^{2}+\xi_{z}^{2}\right) \mathrm{v}_{\xi}, G_{1}=J\left(\eta_{r}^{2}+\eta_{z}^{2}\right) u_{\eta}, G_{2}=J\left(\eta_{r}^{2}+\eta_{z}^{2}\right) \mathrm{v}_{\eta}, \\
& \vec{S}=\left\|\begin{array}{l}
S_{1} \\
S_{2}
\end{array}\right\|+\left\|\begin{array}{l}
S_{r} \\
S_{z}
\end{array}\right\|, \\
& S_{1}=\frac{1}{J} \frac{\partial\left\{J\left(\xi_{r} \eta_{r}+\xi_{z} \eta_{z}\right) u_{\eta}\right\}}{\partial \xi}+\frac{1}{J} \frac{\partial\left\{J\left(\eta_{r} \xi_{r}+\eta_{z} \xi_{z}\right) u_{\xi}\right\}}{\partial \eta}+\alpha \frac{1}{r}\left\{\xi_{r} \frac{\partial u}{\partial \xi}+\eta_{r} \frac{\partial u}{\partial \eta}\right\}
\end{aligned}
$$




$$
\begin{gathered}
S_{2}=\frac{1}{J} \frac{\partial\left\{J\left(\xi_{r} \eta_{r}+\xi_{z} \eta_{z}\right) v_{\eta}\right\}}{\partial \xi}+\frac{1}{J} \frac{\partial\left\{J\left(\eta_{r} \xi_{r}+\eta_{z} \xi_{z}\right) v_{\xi}\right\}}{\partial \eta}+\alpha \frac{1}{r}\left\{\xi_{r} \frac{\partial v}{\partial \xi}+\eta_{r} \frac{\partial v}{\partial \eta}\right\}, \\
S_{r}=(\lambda+\mu)\left[\xi_{r} \frac{\partial\left(\alpha \frac{u}{r}\right)}{\partial \xi}+\eta_{r} \frac{\partial\left(\alpha \frac{u}{r}\right)}{\partial \eta}\right], S_{z}=(\lambda+\mu)\left[\xi_{z} \frac{\partial\left(\alpha \frac{u}{r}\right)}{\partial \xi}+\eta_{z} \frac{\partial\left(\alpha \frac{u}{r}\right)}{\partial \eta}\right],
\end{gathered}
$$

где $u(r, z, t)=U_{r}, v(r, z, t)=U_{z}-$ проекции вектора перемещения $\vec{U}(r, z, t)$ на оси $r$ и $z$; $U_{\xi}=\xi_{r} u+\xi_{z} v, U_{\eta}=\eta_{r} u+\eta_{z} v-$ контравариантные компоненты вектора $\vec{U}$, описывающего перемещения точки тела в криволинейной системе координат $\xi, \eta ; \alpha=0$ - соответствует плоскому и $\alpha=1$ - осесимметричному случаям деформаций.

Теперь преобразуем, используя переход к криволинейной системе координат $\xi, \eta$, уравнение, связанное с переносом внутренней энергии процессом теплопроводности

$$
\begin{gathered}
\rho c_{\varepsilon=0} \frac{\partial \theta}{\partial t}=\frac{1}{J} \frac{\partial\left(J \lambda_{q}\left(\xi_{r}^{2}+\xi_{z}^{2}\right) \theta_{\xi}\right)}{\partial \xi}+\frac{1}{J} \frac{\partial\left(J \lambda_{q}\left(\eta_{r}^{2}+\eta_{z}^{2}\right) \theta_{\eta}\right)}{\partial \eta}+f, \\
S_{\lambda}=\frac{1}{J} \frac{\partial\left\{J \lambda_{q}\left(\xi_{r} \eta_{r}+\xi_{z} \eta_{z}\right) \theta_{\eta}\right\}}{\partial \xi}+\frac{1}{J} \frac{\partial\left\{J \lambda_{q}\left(\eta_{r} \xi_{r}+\eta_{z} \xi_{z}\right) \theta_{\xi}\right\}}{\partial \eta}+\alpha \frac{\lambda_{q}}{r}\left\{\xi_{r} \frac{\partial \theta}{\partial \xi}+\eta_{r} \frac{\partial \theta}{\partial \eta}\right\}, \\
D_{\lambda}=-\alpha_{T}(3 \lambda+2 \mu) T_{0}\left[\frac{1}{J} \frac{\partial J\left(\partial U_{\xi} / \partial t\right)}{\partial \xi}+\frac{1}{J} \frac{\partial J\left(\partial U_{\eta} / \partial t\right)}{\partial \eta}\right]-\alpha \frac{\alpha_{T}(3 \lambda+2 \mu) T_{0} u}{r},
\end{gathered}
$$

где $f=S_{\lambda}+D_{\lambda}$.

\section{3. Отдельные математические детали численного метода расчета}

При численной реализации уравнений (2), (3) в параметрической области $\Omega_{p}$ вводится прямоугольная сетка $\omega_{h}=\left\{\xi_{j}, \eta_{k} ; j=\overline{0, J}, k=\overline{0, K}\right\}, \quad \omega_{h} \in \Omega_{h}$. Здесь $h_{\xi}=\xi_{j}-\xi_{j-1}$, $\xi_{j+1 / 2}=\xi_{j}+0.5 h_{\xi}, \xi_{j-1 / 2}=\xi_{j}-0.5 h_{\xi}, h_{\eta}=\eta_{k}-\eta_{k-1}, \eta_{k+1 / 2}=\eta_{k}+0.5 h_{\eta}, \eta_{k-1 / 2}=\eta_{k}-0.5 h_{\eta}$.

Численное решение системы уравнений (2), (3) осуществляется в два этапа. Сначала на первом этапе неявно разрешается уравнение теплопроводности специального вида (3). Затем на втором этапе находится решение уравнений термоупругости (2).

При решении “тепловой” части двумерных уравнений квазистатической термоупругости, которые описывают перенос внутренней энергии процессом теплопроводности, применяется следующая двухшаговая разностная схема (с использованием работы [11])

$$
\begin{gathered}
c_{j, k} \frac{\theta_{j, k}^{n+1 / 2}-\theta_{j, k}^{n}}{\Delta / 2}=\frac{a_{j+1 / 2, k}\left(\theta_{j+1, k}^{n+1 / 2}-\theta_{j, k}^{n+1 / 2}\right)-a_{j-1 / 2, k}\left(\theta_{j, k}^{n+1 / 2}-\theta_{j-1, k}^{n+1 / 2}\right)}{h_{\xi}^{2}}+F_{\xi}^{n}+O\left(h_{\xi}^{2}, h_{\eta}^{2}\right), \\
\theta=\theta\left(\xi, \eta, t=t^{n}\right), F_{\xi}=\frac{\partial\left[J \lambda_{q}\left(\eta_{r}^{2}+\eta_{z}^{2}\right) \theta_{\eta}\right]}{\partial \eta}+J f, \\
c_{j, k} \frac{\theta_{j, k}^{n+1}-\theta_{j, k}^{n+1 / 2}}{\Delta / 2}=\frac{a_{j, k+1 / 2}\left(\theta_{j, k+1}^{n+1}-\theta_{j, k}^{n+1}\right)-a_{j, k-1 / 2}\left(\theta_{j, k}^{n+1}-\theta_{j, k-1}^{n+1}\right)}{h_{\eta}^{2}}+F_{\eta}^{n+1 / 2}+O\left(h_{\xi}^{2}, h_{\eta}^{2}\right), \\
\theta=\theta\left(\xi, \eta, t=t^{n}+\frac{\Delta t}{2}\right), F_{\eta}=\frac{\partial\left[J \lambda_{q}\left(\xi_{r}^{2}+\xi_{z}^{2}\right) \theta_{\xi}\right]}{\partial \xi}+J f,
\end{gathered}
$$


где $n$ - верхний индекс, относящийся к моменту “времени” $t=n \Delta t, \Delta t$ - шаг по “времени”; $c=\rho c_{\varepsilon=0} J ; a_{\xi}=J \lambda_{q}\left(\xi_{r}^{2}+\xi_{z}^{2}\right) ; a_{\eta}=J \lambda_{q}\left(\eta_{r}^{2}+\eta_{z}^{2}\right)$. Данная разностная схема (вдоль пространственных направлений $\xi, \eta$ ) легко разрешается скалярной прогонкой.

Для решения уравнений термоупругости $A \vec{U}=0$ используем метод установления [12]. При этом шаг по “времени" $\Delta t$ найдем с помощью итерационного метода вариационного типа. Для этого определим вектор невязок $\vec{R}=\left(R_{1}, R_{2}\right)$

$$
\begin{gathered}
R_{1, j, k}^{n}=\left(A U_{j, k}\right)_{1}^{n}-b_{1, j, k}^{n}, R_{2, j, k}^{n}=\left(A U_{j, k}\right)_{2}^{n}-b_{2, j k}^{n}, \vec{b}_{j, m}^{n}=\left\{b_{1, j, k}^{n}, b_{2, j, k}^{n}\right\}=\{0,0\}, \\
j=\overline{1, J}-1, \quad k=\overline{1, K-1}
\end{gathered}
$$

Введем скалярное произведение следующим образом [11]:

$$
\begin{gathered}
(\vec{a}, \vec{b})=\sum_{j=1}^{J-1} \sum_{k=1}^{K-1}\left(a_{1, j, k} b_{1, j, k} h_{\xi} h_{\eta}\right)+\sum_{j=1}^{J-1} \sum_{k=1}^{K-1}\left(a_{2, j, k} b_{2, j, k} h_{\xi} h_{\eta}\right), \\
\vec{a}_{j, k}=\left(a_{1}, a_{2}\right)_{j, k}, \vec{b}_{j, k}=\left(b_{1}, b_{2}\right)_{j, k}
\end{gathered}
$$

Будем минимизировать значение невязки $\vec{R}_{j, k}=A \vec{U}_{j, k}-\vec{b}_{j, k}$, используя модифицированный вариант итерационного метода вариационного типа - метод минимальных невязок [13]. В этом случае итерации следует проводить по формулам

$$
\vec{U}_{j, k}^{n+1}=\vec{U}_{j, k}^{n}+c \Delta t \vec{R}_{j, k}^{n}, c \approx 0.9, \Delta t=\frac{(\vec{R}, A \vec{R})}{(A \vec{R}, A \vec{R})}
$$

При численной реализации метода минимальных невязок для аппроксимации производных от векторов $\vec{F}, \vec{G}, \vec{I}, \vec{W}$ и переменных $u, v, \theta$ зададим на введенной выше сетке $\omega_{h} \in \Omega_{h}$ следующее конечно-разностное представление производных [14]:

$$
\begin{gathered}
\frac{\partial}{\partial \xi}\left[a \frac{\partial g}{\partial \xi}\right]_{j, k}=\frac{a_{j+1 / 2, k}\left(g_{j+1, k}-g_{j, k}\right)-a_{j-1 / 2, k}\left(g_{j, k}-g_{j-1, k}\right)}{h_{\xi}^{2}}+O\left(h_{\xi}^{2}\right), a_{j \pm 1 / 2, k}=\frac{a_{j, k}+a_{j \pm 1, k}}{2}, \\
\frac{\partial}{\partial \xi}\left[a \frac{\partial g}{\partial \eta}\right]_{j, k}=\frac{a_{j+1, k}\left(g_{j+1, k+1}-g_{j+1, k-1}\right)-a_{j-1, k}\left(g_{j-1, k+1}-g_{j-1, k-1}\right)}{4 h_{\xi} h_{\eta}}+O\left(h_{\xi}^{2}, h_{\eta}^{2}\right)
\end{gathered}
$$

Отметим, что для корректной работы описанного алгоритма необходимо:

- $\quad$ после каждого расчетного шага (т.к. произошла деформация границ расчетной области $\Omega$ ) осуществить перестроение расчетной сетки (например: с использованием метода работы [9]);

- $\quad$ затем произвести интерполяцию (с учетом выполнения законов сохранения и нужной степени гладкости решения, см. [15]) величин $\vec{U}(r, z, t)$ и $\theta(r, z, t)$, известных на расчетной сетке в момент времени $t^{n}$ на сетку, полученную после её перестроения.

\section{4. Некоторые результаты расчета}

Для проверки работоспособности сформулированной численной методики была решена группа тестовых задач. Во всех сериях расчетов использовалась система уравнений линейной теории упругости и уравнение теплопроводности в эйлеровых координатах.

Первой тестовой (валидационной) задачей является нахождение равновесного термодеформируемого состояния стальной балки, которая с двух сторон закреплена с помощью неподвижных шарниров (рис. 1) и находится в однородном поле температуры 
$T(x, y) \equiv T_{0}+\Delta T$ (где $T_{0}$ - начальное значение температуры, $\Delta T$ - величина равномерного температурного нагрева). Решается только задача механики в заданном равномерно распределенном температурном поле $T(x, y) \equiv T_{0}+\Delta T$. Точное решение плоской задачи теории термоупругости для прямоугольной полосы выглядит следующим образом [3]:

$$
U_{y}(x)=\frac{h}{2}\left[\frac{\cos (\lambda x)}{\cos (\lambda l / 2)}-1\right], \lambda=\sqrt{\frac{P}{D}}, D=\frac{E h^{3}}{12},
$$

где $h$ - высота балки; $l$ - длина балки; $E$ - модуль упругости первого рода. Сила реакции опор $P$ вычисляется из уравнения

$$
\frac{P \cdot l}{h \cdot E}+\frac{h^{2}}{2} \lambda \cdot \operatorname{tg}\left(\frac{\lambda \cdot l}{2}\right)+\frac{h^{2}}{8} \cdot \frac{P}{D} \cdot \frac{\frac{l}{2}-\frac{\sin (\lambda \cdot l)}{2 \cdot \lambda}}{\cos ^{2}\left(\frac{\lambda \cdot l}{2}\right)}-\alpha \cdot \Delta T \cdot l=0,
$$

где $\alpha$ - коэффициент температурного расширения.

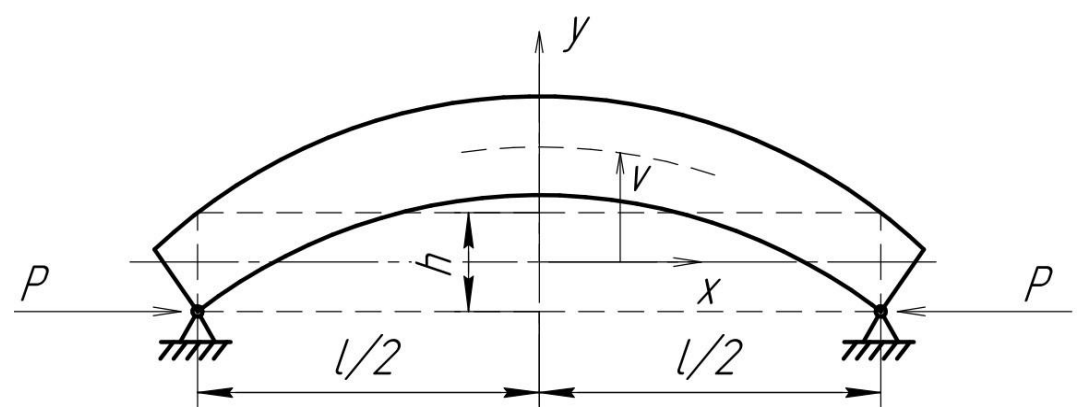

Рис. 1. Схема тестовой задачи об изгибе равномерно нагретой закрепленной балки под действием температурных напряжений

На рис. 2 приведены результаты (при $h=2.5 \mathrm{мm}, l=100 \mathrm{мм}, E=2 \times 10^{11}$ Па, $\alpha=1.2 \times 10^{-5} \mathrm{C}^{-1}$, $\Delta T=36^{\circ} \mathrm{C}$ ) численного расчета первой тестовой задачи по определению прогиба равномерно нагретой закрепленной балки под действием температурных напряжений (максимальное значение относительной ошибки составило $2 \%$ ). $X$, мм

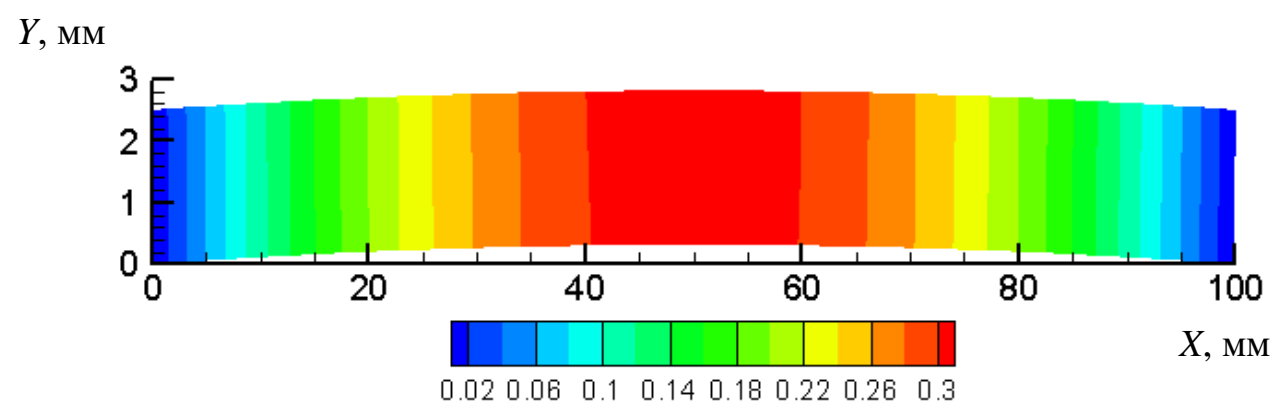

Рис. 2. Результаты численного расчета тестовой задачи по определению прогиба равномерно нагретой закрепленной балки под действием температурных напряжений

Из систематических расчетов известно $[16,17]$, что конвективный тепловой поток $q_{w}$ вблизи передней критической точки можно определить с помощью формул $(R-$ радиус затупления) 


$$
\begin{gathered}
q_{w}=1.93 \times 10^{-4} V_{\infty}^{1.08}\left(H_{0}-H_{w}\right) \sqrt{\frac{\rho_{\infty}}{R}} \text { (в ламинарном случае); } \\
q_{w}=4.69 \times 10^{-4} V_{\infty}^{1.25}\left(H_{0}-H_{w}\right)\left(1+\frac{T_{w}}{T_{0}}\right)^{-2 / 3} \frac{\rho_{\infty}^{0.8}}{R^{0.2}} \text { (в турбулентном случае), }
\end{gathered}
$$

где $H_{w}\left[\right.$ [ж/кг], $T_{w}[\mathrm{~K}]$ - энтальпия, температура на поверхности обтекаемого тела; $H_{0}$ [Дж/кг], $T_{0}[\mathrm{~K}]$ - энтальпия, температура, взятые на внешней границе пограничного слоя; $\rho_{\infty}\left[\kappa г / \mathrm{M}^{3}\right], V_{\infty}$ [м/с] - плотность и скорость невозмущенного натекающего на тело потока; $R[\mathrm{M}]$ радиус тела в передней критической точке; $q_{w}\left[\mathrm{BT} / \mathrm{M}^{2}\right]$ тепловой поток в передней критической точке.

Из данных формул следует, что коэффициент теплоотдачи $\alpha$ в передней критической точке можно оценить с помощью соотношения

$$
\alpha \sim 1 / \sqrt{R} \text { (в турбулентном случае } \alpha \sim 1 / R^{0.2} \text { ) }
$$

Поэтому при больших скоростях движения (M > 6) и, соответственно, больших температурах торможения при уменьшении радиуса затупления $R$ в критической точке резко возрастают (если не учитывать обратного воздействия на конвективный тепловой поток $q_{w}$ роста температуры $T_{w}$ поверхности тела) значения конвективных и радиационных потоков. Данные формулы также демонстрируют заметное влияние на конвективный тепловой поток $q_{w}$ температуры поверхности $T_{w}$ элемента ЛА.

Выполненные в данной работе расчеты (рис. 3-10) позволяют провести оценку воздействия на конвективный тепловой поток $q_{w}$ радиуса затупления $R$, геометрической формы, конструкционного материала и распределения температуры поверхности $T_{w}$ элемента ЛА.

Для валидации и верификации разработанной численной методики в качестве второй тестовой задачи были взяты данные работ $[18,19]$. Геометрия (рис. 3-5) тестирующей задачи представляет собой $2 D$ клинообразную оболочку (материал оболочки - сплав Inconel 617, длина клина 7.62 мм, толщина оболочки постоянна и равна 11 мм, угол раскрытия клина $6^{\circ}$ ), затупленную по цилиндру (радиус затупления $R=3.18$ мм).

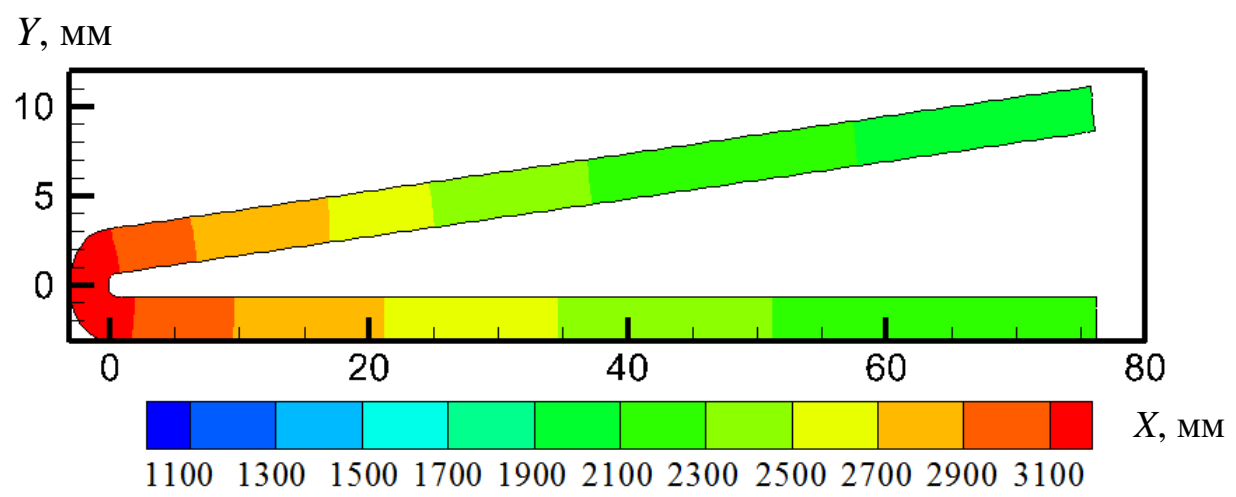

Рис. 3. Распределение температуры $T[\mathrm{~K}]$ в клинообразной оболочке (сплав Inconel 617), затупленной по цилиндру. Радиус затупления цилиндра $R=3.18$ мм, число Маха в набегающем потоке $\mathrm{M}=5.25$

Конвективный поток тепла, поступающий на поверхность клинообразной оболочки, а также аэродинамические нагрузки, распределенные вдоль её поверхности, взяты из работ $[18,19]$. Параметры потока воздуха, натекающего на элемент ЛА (кромки крыльев, носового обтекателя), определяются числом Маха $\mathrm{M}=5.25$. Принимается, что стабилизация внутренней энергии в элементе ЛА, который жестко заделан, осуществляется тепловым излучением. 


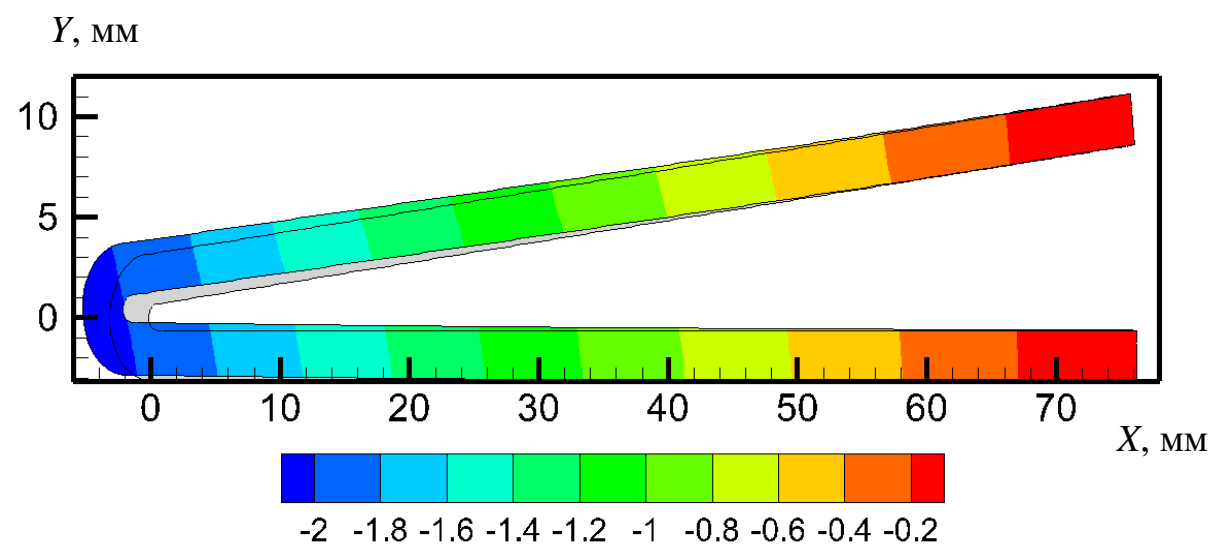

Рис. 4. Распределение перемещений $U_{x}[\mathrm{Mм}]$ в направлении оси $X$ в клинообразной оболочке (сплав Inconel 617), затупленной по цилиндру. Радиус затупления цилиндра $R=3.18$ мм, число Маха в набегающем потоке $M=5.25$. Сплошными линиями указаны контуры исходного недеформированного тела

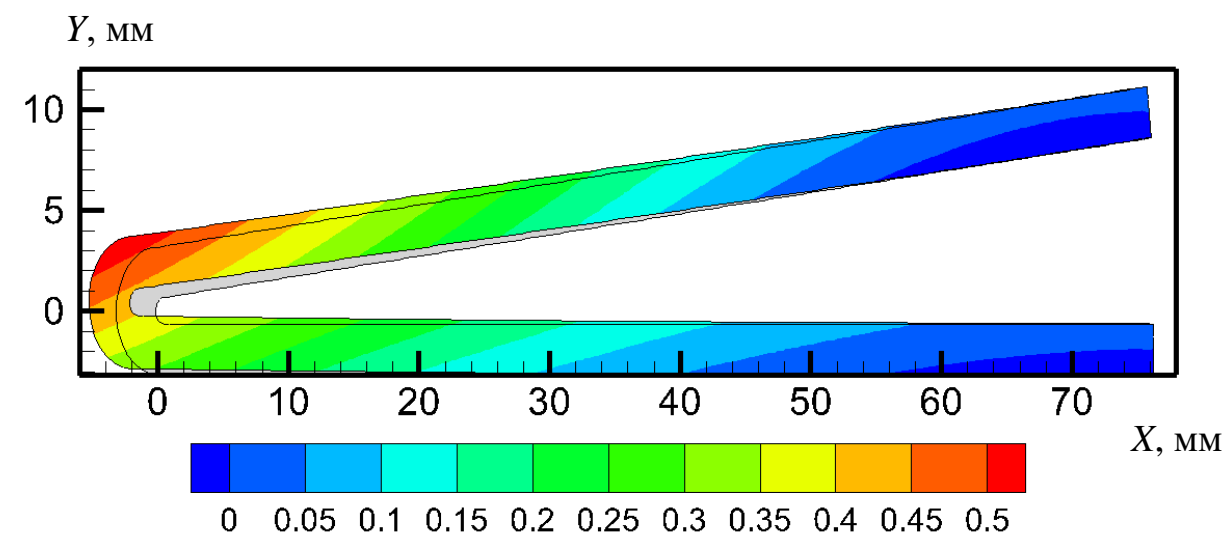

Рис. 5. Распределение перемещений $U_{y}$ [мм] в направлении оси $Y$ в клинообразной оболочке (сплав Inconel 617), затупленной по цилиндру. Радиус затупления цилиндра $R=3.18$ мм, число Маха в набегающем потоке $M=5.25$. Сплошными линиями указаны контуры исходного недеформированного тела

Результаты расчета равновесного термодеформируемого состояния, соответствующие второй тестовой задаче (рис. 3-5), показывают:

- $\quad$ максимальное значение относительной ошибки, как по распределению температуры, так и по значениям перемещений, составляет величину $<3 \%$;

- $\quad$ имеется заметное изменение распределения температуры $T_{w}$ вдоль поверхности элемента ЛА $T_{w}=1100 \div 3100 \mathrm{~K}$;

- $\quad$ перемещения $U_{x}$ передней части элемента ЛА в направлении оси $X$ равны 2 мм в сторону удлинения тела;

- $\quad$ перемещения $U_{y}$ передней части элемента ЛА в направлении оси $Y: 0.25$ мм - в нижней части тела, 0.5 мм - в верхней части тела;

- $\quad$ радиус затупления $R$ изменяется и достигает величины $R=2.10$ мм (приводя к возрастанию конвективного потока тепла $q_{w}$ на $23 \%$ ), что на $34 \%$ меньше исходного радиуca $R=3.18$ мм.

Следующая задача состояла в нахождении распределения температуры $T$ и перемещений $\vec{U}$ в элементе конструкции ЛА - в цилиндре, затупленном по сфере. При решении этой задачи была задана следующая геометрия элемента ЛА (сферическое затупление ра- 
диуса 1.27 см, длина цилиндра 10 см), а также конвективный поток тепла (рис. 6), падающий на поверхность рассматриваемого элемента.

В качестве газодинамических расчетных данных в набегающем внешнем потоке, использовались данные работы [20]: давление $P=0.23 \times 10^{3}$ Па; плотность $\rho=0.178 \times 10^{-5} \mathrm{r} / \mathrm{cm}^{3}$; скорость $V=4.167 \times 10^{5} \mathrm{~cm} / \mathrm{c}$; температура $T=450 \mathrm{~K}$. При этом температура поверхности элемента считалась постоянной и равной $T_{w}=300 \mathrm{~K}$. Распределение плотности конвективного теплового потока вдоль обтекаемой некаталитической поверхности взято из работы [18] и показано на рис. 6. На рис. 7. приведено распределение температуры газа вдоль поверхности цилиндра, затупленного по сфере [21-32]. Отметим, что указанные аэротермофизические значения соответствуют гиперзвуковому режиму течения газа $(M=9.8)$.

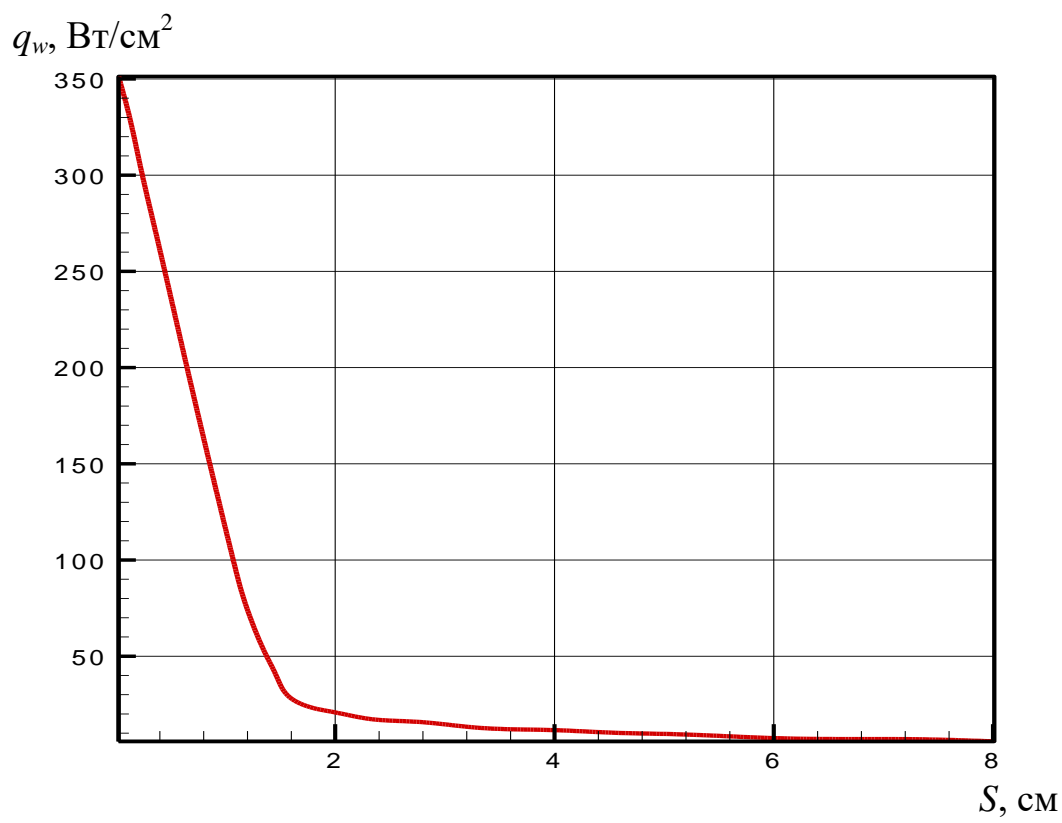

Рис. 6. Распределение плотности конвективного теплового потока вдоль поверхности цилиндра затупленного по сфере [20]. Радиус затупления $R=1.27$ см

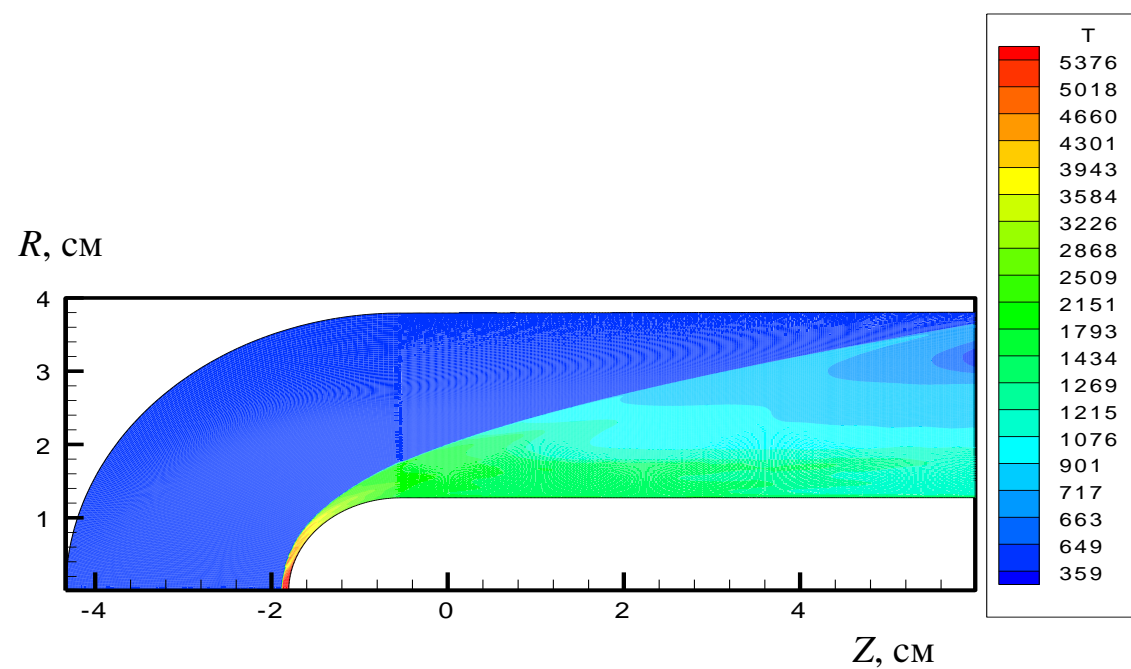

Рис. 7. Распределение температуры $T[\mathrm{~K}]$ вдоль поверхности цилиндра, затупленного по сфере. Радиус затупления сферы $R=1.27$ см, скорость набегающего потока $V_{\infty}=4.17 \mathrm{kм} / \mathrm{c}$ (число Maха $\mathrm{M}=9.8$, высота $h=22$ км) 
При решении данной задачи так же, как и раньше принимается, что потери внутренней энергии элементом ЛА осуществляется только за счет теплового излучения. Сам элемент ЛА (рис. 8-10) выполнен из вольфрама и жестко заделан.

На рис. 8-10 приведены результаты расчетов, выполненных с помощью вышеприведенной методики для рассматриваемого элемента ЛА.

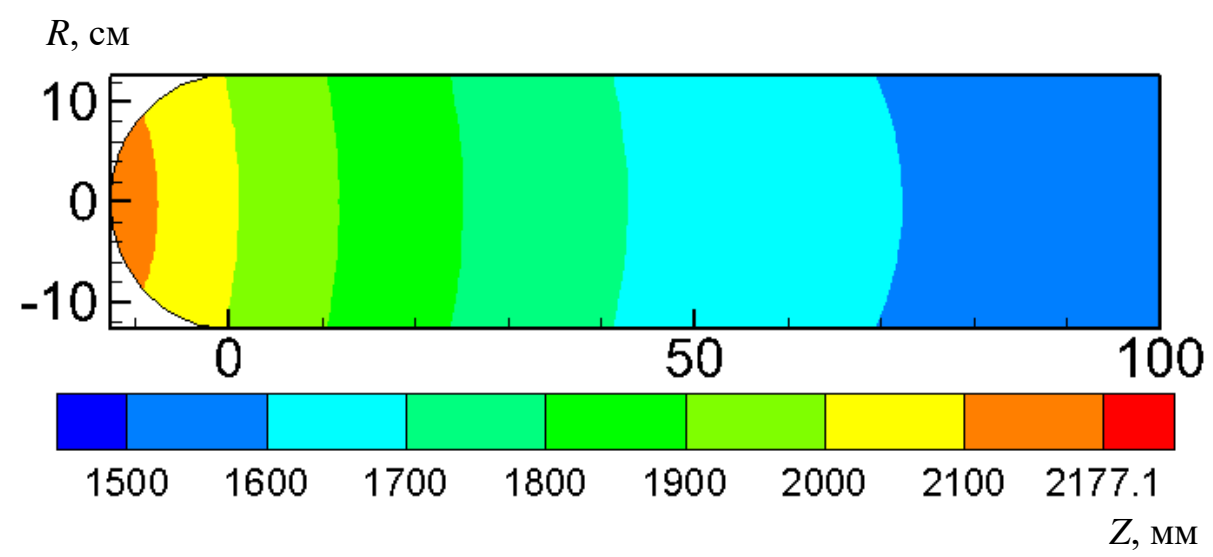

Рис. 8. Распределение температуры $T[\mathrm{~K}]$ в затупленном по сфере цилиндре. Радиус затупления сферы $R=1.27$ см, скорость набегающего потока $V_{\infty}=4.17$ км $/$ с (число Маха $M=9.8$, высота $h=22$ км)

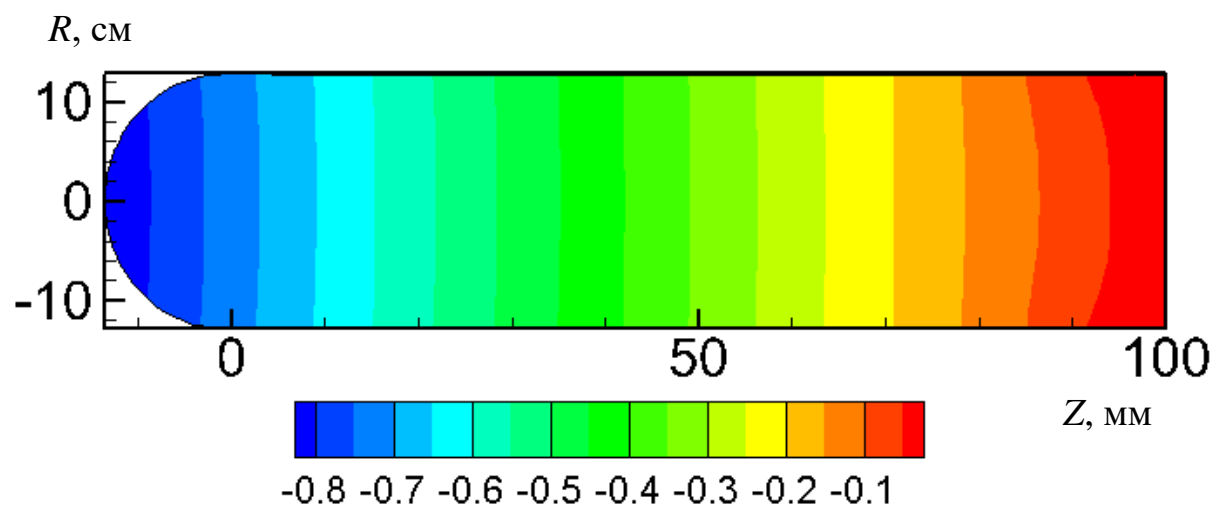

Рис. 9. Распределение перемещений $U_{x}$ [мм] в направлении оси $R$ в затупленном по сфере цилиндре. Радиус затупления сферы $R=1.27$ см, скорость набегающего потока $V_{\infty}=4.17$ км/с (число Маха $\mathrm{M}=9.8$, высота $h=22$ км)

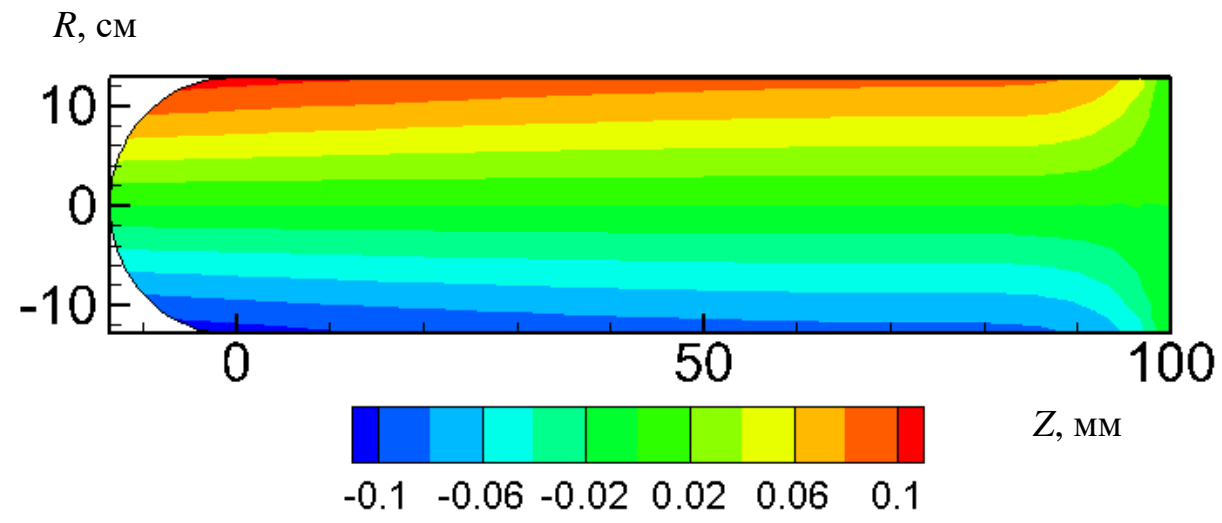

Рис. 10. Распределение перемещений $U_{z}$ [мм] в направлении оси $Z$ в затупленном по сфере цилиндре. Радиус затупления сферы $R=1.27 \mathrm{~cm}$, скорость набегающего потока $V_{\infty}=4.17$ км/с (число Маха $M=9.8$, высота $h=22$ км) 
Из приведенных графических зависимостей рис. 8-10 следует:

- $\quad$ распределение температуры поверхности $T_{w}=1500 \div 2200 \mathrm{~K}$, обтекаемого внешним потоком элемента ЛА (вдоль образующей элемента ЛА), отличается от постоянного и существенно превышает температуру $\left(T_{w}=300 \mathrm{~K}\right)$ поверхности элемента, принятую в качестве граничного условия при расчете аэротермодинамики ЛА;

- $\quad$ радиус затупления $R$ изменяется на $4.6 \%$ (с $R=12.7$ мм до значений $R=12.12$ мм). При этом конвективный поток тепла $q_{w}$ в передней критической точке за счет изменения радиуса кривизны $R$ возрастает на $2.4 \%$. Однако, если учесть существенный рост температуры поверхности $T_{w}=2200 \mathrm{~K}$, то изменение будет уже на уровне $q_{w} \approx 10 \div 20 \%$;

- $\quad$ перемещения $\vec{U}$ точек рассматриваемого сплошного элемента ЛА невелики и, в основном, концентрируются вблизи заделки;

- $\quad$ конвективный тепловой поток (см. рис. 6), взятый из работы [20], должен быть скорректирован на 70 \% вдоль образующей цилиндра при учете решений уравнений квазистатической термоупругости (из-за существенного повышения температуры в теле и на поверхности элемента ЛА).

\section{6. Заключение}

Сформулирована $2 D$ расчетно-теоретическая методика, позволяющая находить решение системы уравнений термоупругости с граничными условиями общего вида. Проведена первоначальная валидация и верификация разработанной численной методики на основе тестовой задачи. Выполнено численное моделирование температурных полей и термонапряжений в простейших элементах конструкций летательных аппаратов (клинообразная оболочка, затупленная по цилиндру, цилиндр, затупленный по сфере). Из выполненных расчетов следует, что численное моделирование физических процессов, протекающих на поверхности (и в ближайшей окрестности) высокоскоростных летательных аппаратов, необходимо проводить на основе комплексных, взаимно согласованных, сопряженных математических моделей аэротермодинамики, теплообмена и термопрочности. Отметим также, что кромки корпуса, крыльев и носового обтекателя корпуса целесообразно (в этом случае деформации будут минимальны) выполнять в виде цельной детали.

\section{Литература}

1. Карташов Э.М. Аналитические методы в теории теплопроводности твердых тел. Изд. 3-е, перераб. и доп. М.: Высшая школа, 2001. 550 с.

2. Димитриенко Ю.И., Захаров А.А., Коряков М.Н., Сыздыков Е.К., Минин В.В. Численное решение сопряженной задачи гиперзвуковой аэродинамики и термомеханики термодеструктирующих конструкций // Инженерный журнал: наука и инновации, 2013, вып. 9. URL: http://engjournal.ru/catalog/mathmodel/aero/1114.html

3. Коваленко А.Д. Термоупругость. Учебное пособие. К.: Вища школа, 1975. 215 с.

4. Димитриенко Ю.И., Захаров А.А., Коряков М.Н., Сыздыков Е.К. Моделирование сопряженных процессов аэрогазодинамики и теплообмена на поверхности теплозащиты перспективных гиперзвуковых летательных аппаратов // Известия высших учебных заведений. Машиностроение. 2014. № 3 (648). С. 23-34.

5. Кузенов В.В., Рыжков С.В. Радиационно-гидродинамическое моделирование контактной границы плазменной мишени, находящейся во внешнем магнитном поле // Прикладная физика. 2014. № 3. С. 26-30.

6. Котов М.А., Кузенов В.В. Численное моделирование обтекания поверхностей перспективных гиперзвуковых летательных аппаратов // Вестник МГТУ им. Н.Э. Баумана. Сер. "Машиностроение". 2012. № 3. С. 17-30. 
7. Ryzhkov S.V. Compact toroid and advanced fuel - together to the Moon?! // Fusion Science and Technology. 2005. V. 47, № 1T. Pp. 342-344.

8. Рыжков С.В. Моделирование теплофизических процессов в магнитном термоядерном двигателе // Тепловые процессы в технике. 2009. № 9. С. 397-400.

9. Кузенов В.В. Построение регулярных адаптивных сеток в пространственных областях с криволинейными границами // Вестник МГТУ им. Н.Э. Баумана. Сер. “Машиностроение”. 2008. №1. С. 3-11.

10. Андерсон Д., Таннехилл Дж., Плетчер Р. Вычислительная гидромеханика и теплообмен: В 2-х т. Т. 1: Пер. с англ. М.: Мир, 1990. 384 с.

11. Самарский А.А. Введение в теорию разностных схем. М.: Главная редакция физикоматематической литературы изд-ва «Наука», 1971.

12. Самарский А.А., Николаев Е.С. Методы решения сеточных уравнений. М.: Наука, 1978. 590 с.

13. Альшина Е.А., Болтнев А.А., Качер О.А. Эмпирическое улучшение простейших градиентных методов // Математическое моделирование. 2005. Т. 17, № 6. С. 43-57.

14. Головачев Ю.П. Численное моделирование течений вязкого газа в ударном слое. М.: Наука, 1996. $376 \mathrm{c}$.

15. Григорьев Ю.Н., Вшивков В.А., Федорук М.П. Численное моделирование методами частиц в ячейках Рос. акад. наук, Сиб. отд-ние, Ин-т выч. технологий, Новосиб. гос. ун-т. Новосибирск: Изд-во СО РАН, 2004. 360 с.

16. Лунёв В.В. Гиперзвуковая аэродинамика. М.: Машиностроение, 1975. 328 с.

17. Лунёв В.В. Течение реальных газов с большими скоростями. М.: ФИЗМАТЛИТ, 2007. 760 с.

18. Pandey A.K., Dechaumphai P., Weiting A.R. Thermal-Structural Finite Element Analysis using Linear Flux Formulation // NASA Technical Memorandum 102746. 1990. 10 p.

19. Three-Dimensional Thermal Structural Analysis of a Swept Cowl Leading Edge Subjected to Skewed Shock-Shock Interference Heating / S.P. Polesky et al. // Journal of Thermophysics. 1992. V. 6, № 1. Pp. 48-54.

20. Суржиков С.Т. Конвективный нагрев сферического затупления малого радиуса при относительно малых гиперзвуковых скоростях // ТВТ. 2013. Т. 51. С. 261-276.

21. Кузенов В.В. Тестирование отдельных элементов метода расчета физических процессов в мишени магнитно-инерциального термоядерного синтеза // Прикладная физика. 2016. № 2. C. $16-24$.

22. Кузенов В.В., Рыжков С.В. Численное моделирование процесса лазерного сжатия мишени, находящейся во внешнем магнитном поле // Математическое моделирование. 2017. Т. 29. № 9. C. 19-32.

23. Kuzenov V.V., Ryzhkov S.V. Approximate method for calculating convective heat flux on the surface of bodies of simple geometric shapes // Journal of Physics: Conference Series. 2017. V. 815. P. 012024.

24. Кузенов В.В., Лебо А.И., Лебо И.Г., Рыжков С.В. Физико-математические модели и методы расчета воздействия мощных лазерных и плазменных импульсов на конденсированные и газовые среды. 2-е изд. М.: Изд-во МГТУ им. Н.Э. Баумана, 2017. 328 с.

25. Kuzenov V.V., Ryzhkov S.V., Shumaev V.V. Application of Thomas-Fermi model to evaluation of thermodynamic properties of magnetized plasma // Problems of Atomic Science and Technology. 2015. No. 1 (95). Pp. 97-99.

26. Kuzenov V.V., Ryzhkov S.V., Shumaev V.V. Numerical thermodynamic analysis of alloys for plasma electronics and advanced technologies // Problems of Atomic Science and Technology. 2015. No. 4 (98). Pp. 53-56.

27. Ryzhkov S.V., Kuzenov V.V. Analysis of the ideal gas flow over body of basic geometrical shape // International Journal of Heat and Mass Transfer. 2019. V. 132. Pp. 587-592 
28. Kuzenov V.V., Dobrynina A.O., Shumaev V.V. Calculating processes of laminar and turbulent heat transfer around the elements of the aircraft // Journal of Physics: Conference Series. 2018. V. 980. P. 012023.

29. Shumaev V.V., Kuzenov V.V. Development of the numerical model for evaluating the temperature field and thermal stresses in structural elements of aircrafts // Journal of Physics: Conference Series. 2017. V. 891. P. 012311.

30. Суржиков С.Т. Расчетное исследование аэротермодинамики гиперзвукового обтекания затупленных тел на примере анализа экспериментальных данных Москва: ИПМех РАН, 2011. 192 с.

31. Котов М.А., Рулева Л.Б., Солодовников С., Суржиков С.Т. Проведение экспериментов по обтеканию моделей в гиперзвуковой ударной аэродинамической трубе//Физико-химическая кинетика в газовой динамике. 2013. Т. 14, вып. 4. http://chemphys.edu.ru/issues/2013-144/articles/428/

32. Глушко Г.С., Иванов И.Э., Крюков И.А. Моделирование турбулентности в сверхзвуковых струйных течениях // Физико-химическая кинетика в газовой динамике. 2010. Том 9. http://chemphys.edu.ru/issues/2010-9/articles/142/

Статья поступила в редакцию 27 ноября 2018 г. 